\title{
PROBLÈMES POSÉS POUR L'APPLICATION DES ALLIAGES DE NIOBIUM AUX AUBES DE TURBINE
}

\author{
par G. GAUJE et R. BRUNETAUD \\ Direction Technique de la SNECMA
}

\begin{abstract}
Résumé. - L'emploi d'alliages de niobium à la place des alliages classiques base nickel ou cobalt pour la fabrication des parties les plus chaudes des turbomachines représente une solution intéressante aux problèmes posés par l'accroissement des températures de fonctionnement.

L'exposé ci-dessous énumère les principales propriétés requises pour ce genre d'application, décrit les essais de laboratoire correspondants et donne quelques résultats obtenus. Il ressort nettement de cette étude que le point critique est la mise au point d'une protection capable de résister à l'oxydation, notamment en conditions cycliques ainsi qu'aux impacts de corps étrangers. Enfin, des indications sont données sur les progrès à réaliser pour atteindre le stade des essais sur moteur.
\end{abstract}

\begin{abstract}
The use of niobium alloys instead of the current alloys with nickel or cobalt as parent metal for manufacturing the hottest sections of gas turbine engines features an interesting answer to problems introduced by the increase in running temperatures.

The following statement lists the oustanding properties required for this sort of practice, it outlines the relative laboratory tests and specifies some results which were achieved. This research emphasizes clearly that the critical point is the development of a coating which would be capable of resisting to oxidation, especially in cyclical conditions, and to impacts of foreign objects as well. Finally, information is provided concerning improvements to be made in view to reach the trial phase on engines.
\end{abstract}

1. Introduction. - La recherche de températures de combustion élevées dans les turbomachines constitue un fait important dans l'amélioration des performances.

Les alliages classiques base nickel et base cobalt ont évolué progressivement, mais un saut important a été réalisé ces dernières années par l'introduction des techniques de refroidissement.

En utilisant des prélèvements raisonnables d'air au compresseur, de l'ordre de 1 à $1,5 \%$, il est possible d'abaisser la température d'une aube mobile de 100 à $150^{\circ} \mathrm{C}$ par une méthode de refroidissement interne par convection. Des techniques de refroidissement plus évoluées, faisant appel à la sudation ou à la formation d'un film superficiel externe peuvent procurer des gains encore plus importants.

Le refroidissement interne par convection s'applique en général aux aubes fixes et mobiles de $1^{\mathrm{e}^{\mathrm{r}}}$ étage de turbine. Il commence à être nécessaire pour le second étage, mais les pertes de rendement et les complications technologiques qui en résultent deviennent importantes. Si l'on veut éviter cette sujétion, il est néces- saire de disposer d'alliages à performances nettement plus élevées que celles des alliages base nickel ou cobalt limités par leur point de fusion. Parmi les alliages plus réfractaires, ce sont les alliages de niobium qui apparaissent comme étant les plus prometteurs.

2. Introduction des alliages de niobium. - En considérant les courbes des caractéristiques spécifiques de fluage comparées des meilleurs alliages base nickel et d'un alliage de niobium dont nous donnons les compositions ci-dessous, on constate des gains de l'ordre de 120 à $150^{\circ} \mathrm{C}$ dans le domaine de contraintes des aubes mobiles (Fig. 1).

Il s'agit là d'un avantage très net pour des aubes de $2^{\mathrm{e}}$ étage venant après un $1^{\mathrm{e}^{\mathrm{r}}}$ étage refroidi d'un moteur évolué. Nous n'excluons pas l'étape suivante, qui constitue un problème encore plus complexe, à savoir des aubes refroidies en alliage base niobium.

ture risquant d'être au-dessous de la température de transition ductile-fragile du matériau constituant les aubes, le régime d'attente au ralenti qui peut se faire au tiers de la température plein régime, le décollage

\begin{tabular}{|c|c|c|c|c|c|c|c|c|c|c|c|c|}
\hline Alliage & $\mathrm{Ni}$ & $\mathrm{Cr}$ & Co & Mo & $\mathrm{Ti}$ & $\mathrm{Al}$ & $\mathrm{Fe}$ & $\mathrm{Zr}$ & W & V & $\mathrm{Nb}$ & $\mathrm{C}$ \\
\hline INCO $\overline{713}$ & base & $\overline{12 / 14}$ & 1 & $\overline{4,5}$ & $\overline{0,5 / 1}$ & $5, \overline{5 / 6}, 5$ & $\overline{26}$ & $\overline{0,1}$ & - & - & $\overline{2,5}$ & $\overline{0,12}$ \\
\hline IN $100 \ldots$ & base & $8 / 11$ & $13 / 17$ & 3 & $4,5 / 5$ & $5 / 6$ & 1 & 0,05 & & & & 0,15 \\
\hline P $353 \mathrm{M} \ldots \ldots$ & & & & & 0,6 & & & 3,5 & 10 & 2 & base & 0,3 \\
\hline TZM $\ldots . .$. & & & & base & 0,5 & & & 0,1 & & & & 0,03 \\
\hline
\end{tabular}




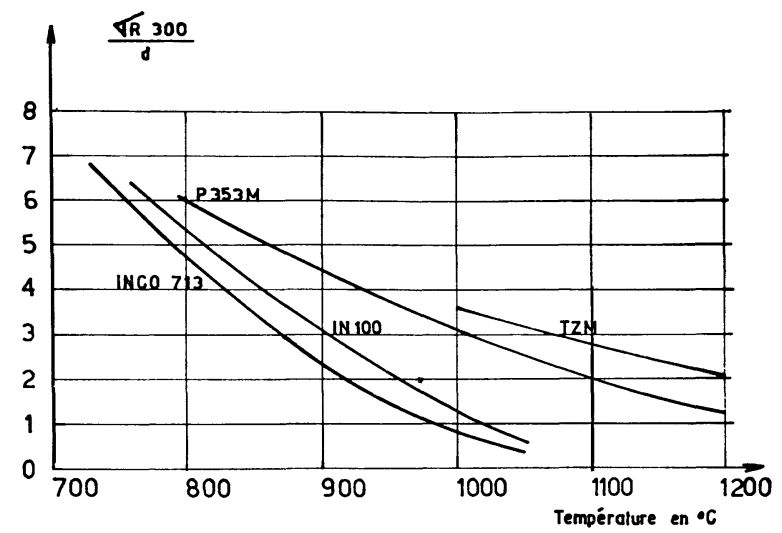

FIG. 1. - Comparaison de contraintes de rupture en $300 \mathrm{~h}$ rapportées à la densité.

Avec les aubages de niobium, le problème majeur est celui de la protection. Pour donner une idée de la difficulté du problème, nous indiquerons qu'un échantillon d'alliage base niobium se trouve réduit en poudre après $24 \mathrm{~h}$ à $700^{\circ} \mathrm{C}$ alors que l'alliage base nickel IN 100 perd environ $25 \mathrm{~g} / \mathrm{m}^{2}$ durant le même temps, mais à $1100^{\circ} \mathrm{C}$ tout en donnant une couche d'oxydes légèrement protecteurs (Fig. 2). Lorsqu'on sait qu'un tel alliage requiert malgré tout une protection en service, par exemple pour des aubes mobiles d'un moteur militaire destinées à fonctionner 300 à $400 \mathrm{~h}$ à $950^{\circ} \mathrm{C}$ sur un potentiel total de $1200 \mathrm{~h}$, on mesure toutes les difficultés de la protection d'aubes en niobium appelées à fonctionner au moins $100^{\circ} \mathrm{C}$ plus haut.

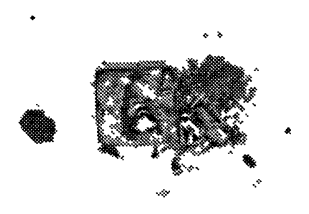

Alliage de niobium nu oxydé 24 heures à $700^{\circ} \mathrm{C}$

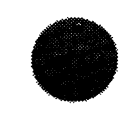

Alliage NK 15 CAT nu oxydé 100 heures à $1100^{\circ} \mathrm{C}$

FIG. 2. - Tenue comparée à l'oxydation d'un alliage de niobium (P $353 \mathrm{M})$ à $700^{\circ} \mathrm{C}$ et d'un alliage base nickel (IN 100) à $1100^{\circ} \mathrm{C}$.

Aubes FIXEs. - Avant de nous attarder sur les aubes mobiles de turbine, nous indiquerons en passant, que les alliages de niobium protégés peuvent également s'envisager pour d'autres pièces telles que des aubes fixes, des éléments de chambres de combustion, des anneaux accroche-flamme de post-combustion, etc... Ces pièces, à la différence des aubes mobiles, sont soumises à des contraintes relativement faibles, mais subissent des effets importants de fatigue thermique; l'intérêt des alliages base niobium est de les supporter assez facilement en raison de leur ductilité à chaud et des pointes de température de l'ordre de $1400^{\circ} \mathrm{C}$, au-delà des points de fusion des alliages base nickel et cobalt, peuvent être envisagées.

3. Conditions de fonctionnement des aubes mobiles de turbine. - Les aubes mobiles sont portées à des températures plus faibles que les fixes. La température est circulairement uniforme par intégration dans le champ tournant, mais les contraintes sont beaucoup plus élevées.

Leur fixation sur les disques pose également des problèmes d'emmanchement plus aigus. Ces fixations sont soumises à des effets de matage et de fatigue, sous contraintes répétées, à des températures limitées pour l'instant à $650-700^{\circ} \mathrm{C}$, par les possibilités des matériaux pour disques.

FluAGE. - Le problème de la tenue en fluage est résumé sur la figure 3 qui donne un exemple de répartition radiale des contraintes et des températures dans une aube mobile. Les contraintes correspondent à une accélération de $40000 \mathrm{~g}$ environ pour un matériau type $\mathrm{P} 353 \mathrm{M}$ dont le poids spécifique est de 8,7. Les caractéristiques moyennes de fluage de cet alliage sont tracées à droite du graphique. La réalisation de la même pièce en alliage base nickel IN 100 n'est possible qu'avec refroidissement; en effet, cet alliage est utilisé actuellement à une température maximale de $950^{\circ} \mathrm{C}$ sous une contrainte de l'ordre de 13 hbar.

Le champ températures-contraintes de la figure 3 n'est donné que pour un cas de vol. Il faut en réalité tenir compte de tous les régimes de fonctionnement moteur : le démarrage par temps froid à une tempéraà pleine charge, l'attente en vol, le régime de croisière, l'arrêt brusque du moteur qui correspond à une

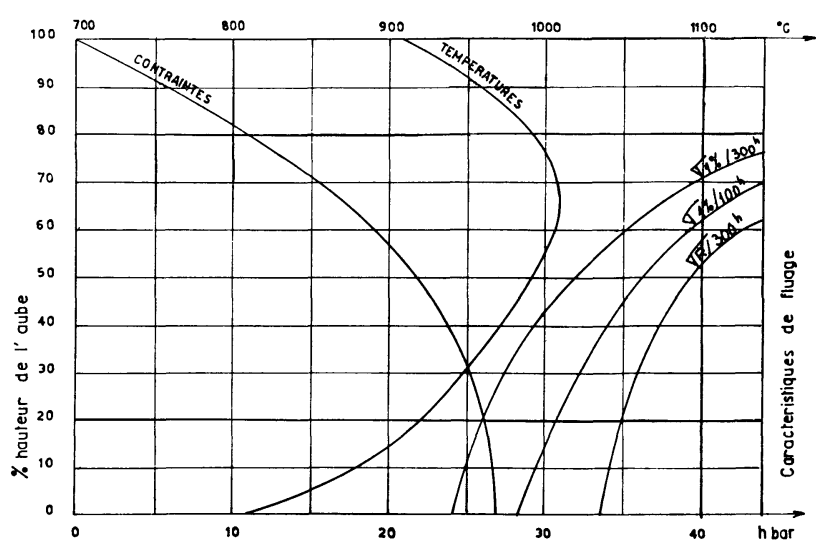

FIg. 3. - Position des caractéristiques de fluage du P 353 M dans un diagramme contraintes ; températures d'une aube mobile. 
trempe à l'air soufflé, etc... On comprend donc qu'il est très difficile de simuler toutes ces conditions et nous aurons l'occasion d'y revenir en parlant des essais de laboratoire.

FATIGUE MÉCANIQUE. - Nous dirons un mot rapide de la fatigue ; elle concerne surtout le pied de l'aube où elle est en fait plus critique que le fluage. On considère en général la fatigue sous entaille avec un coefficient de concentration de contrainte de l'ordre de 2 .

FAtigue thermiQue. - La pale subit en plus du fluage une sollicitation en fatigue thermique lors des variations de régime moteur ou des extinctions. La partie la plus exposée est le bord de fuite dont l'inertie thermique est plus faible que le reste du profil.

OXYDATION. - L'oxydation prend une importance croissante depuis que l'amélioration des alliages réfractaires en fluage a permis d'augmenter la température devant turbine et ceci d'autant plus que cette amélioration correspond à une baisse de la teneur en chrome pour les alliages base nickel. Il en résulte que ceux-ci reçoivent maintenant une couche de protection. Nous verrons plus loin comment ce problème se présente pour les alliages de niobium.

IMPACTS. - Les petits impacts ne doivent pas diminuer de façon notable la durée de vie et en particulier affecter dangereusement la tenue en fatigue et à l'oxydation. Le problème des impacts importants est critique car les utilisateurs considèrent de façon très défavorable tout retour prématuré du moteur en usine. Les durées de vie entre révisions sont de l'ordre de 300 à $1000 \mathrm{~h}$ sur moteur militaire et de plusieurs milliers d'heures sur moteurs civils, avec demande constante d'accroissement des garanties des constructeurs. A titre indicatif, en ce qui nous concerne, sur 30 moteurs revenus en révision avant la date normale, 12 sont rentrés pour impacts sur aube de turbine. Ces 12 moteurs présentaient un ou plusieurs impacts sur le $1^{\mathrm{e}^{\mathrm{r}}}$ étage de turbine et l'un d'entre eux en présentait 2 sur le $2^{\mathrm{e}}$ étage. Les impacts se situent le plus souvent au bord d'attaque ou au bord de fuite, à la périphérie par suite de l'effet de centrifugation dans la turbine.

Les aubes comportant de gros impacts sont remplacées. Celles présentant des petits impacts sont retouchées et il est nécessaire de refaire les protections éventuelles contre l'oxydation. Dans ce problème particulièrement délicat pour les protections des aubes de niobium, il apparaît nécessaire qu'un impact important permette cependant un minimum de durée de vie de quelques heures, dans la mesure où il peut être détecté dans les examens rapides effectués entre 2 vols. Toutefois, une durée de vie de plusieurs centaines d'heures apparaît en fait nécessaire.

PROBLÈME DU BORD DE FUITE. - L'un des problèmes les plus difficiles de l'aube niobium, est la tenue du bord de fuite. Pour des questions de rendement aérody- namique, l'épaisseur nominale de celui-ci est de l'ordre de 0,5 à $1 \mathrm{~mm}$. Pour des aubes qui seraient de grandes dimensions, il serait possible de porter cette épaisseur à $2 \mathrm{~mm}$; dans ces conditions, les pertes sur le rendement global turbine sont encore tolérables. Au-delà de cette valeur, le gain obtenu en température risque d'être compromis, car il faudrait allonger la corde et augmenter le poids des aubes.

ETAT DE SURFACE. - Un dernier problème géométrique, est celui de l'état de surface du pied et de la pale.

Le pied requiert une protection contre l'oxydation mais doit satisfaire à des tolérances dimensionnelles assez serrées, pour lesquelles des dépôts assez lisses, du type phase vapeur doivent bien convenir.

La pale pourra admettre des tolérances plus larges et une certaine rugosité acceptable du point de vue aérodynamique. Des dépôts obtenus à partir de poudres en suspension dans un liant, déposées par projection ou au trempé, avec traitement thermique ultérieur, peuvent être admis dans ce cas.

4. Essais des alliages niobium nus ou protégés. Nous venons de passer en revue les sollicitations auxquelles est soumise une aube mobile de turbine, en posant d'une façon plus précise le cas d'une aube qui serait fabriquée en alliage de niobium revêtu.

Avant de faire tourner de telles pièces sur un banc de turbine, il est nécessaire, compte tenu du prix très élevé d'un tel essai, d'approcher le plus près possible de la solution par des essais en laboratoire, d'une part sur matériau non protégé pour définir ses propriétés intrinsèques, d'autre part sur matériau revêtu pour évaluer ses propriétés en conditions réelles.

4.1 CARACTÉRISTIQUES MÉCANIQUES SUR MATÉRIAU SANS PROTECTION. - Traction. - Il conviendra de chiffrer, la résistance à la traction, à l'ambiante à l'air sur éprouvettes lisses et entaillées pour vérifier que la résistance et la ductilité sont suffisantes. Ensuite il faudra chiffrer les caractéristiques $R, E, A \%, \Sigma \%$ dans le domaine d'emploi envisagé soit ici de $700^{\circ}$ à $1100^{\circ} \mathrm{C}$. Ces mesures seront effectuées sous vide. Il faudra veiller à ce qu'il n'y ait pas de valeurs de ductilité anormalement faibles.

Fluage. - Pour la caractérisation du matériau en fluage les essais sont également effectués sous vide. En fluage allongement nous donnerons, à titre indicatif, 2 valeurs repères situées aux limites du domaine d'emploi envisagé plus haut, et qui serviront, dans une première approche à sélectionner les alliages :

- $780^{\circ} \mathrm{C} / 40$ hbar, a $\%<1 \%$ en $150 \mathrm{~h}$.

$-1080^{\circ} \mathrm{C} / 18 \mathrm{hbar}, \mathrm{a} \%<1 \%$ en $150 \mathrm{~h}$.

Compte tenu de notre expérience sur d'autres matériaux, les essais de fluage rupture devront conduire, à des allongements de rupture d'au moins $5 \%$ et à des strictions supérieures à $10 \%$. L'une des machines 
d'essais mécaniques sous vide adaptée pour ces mesures est représentée à la figure 4.

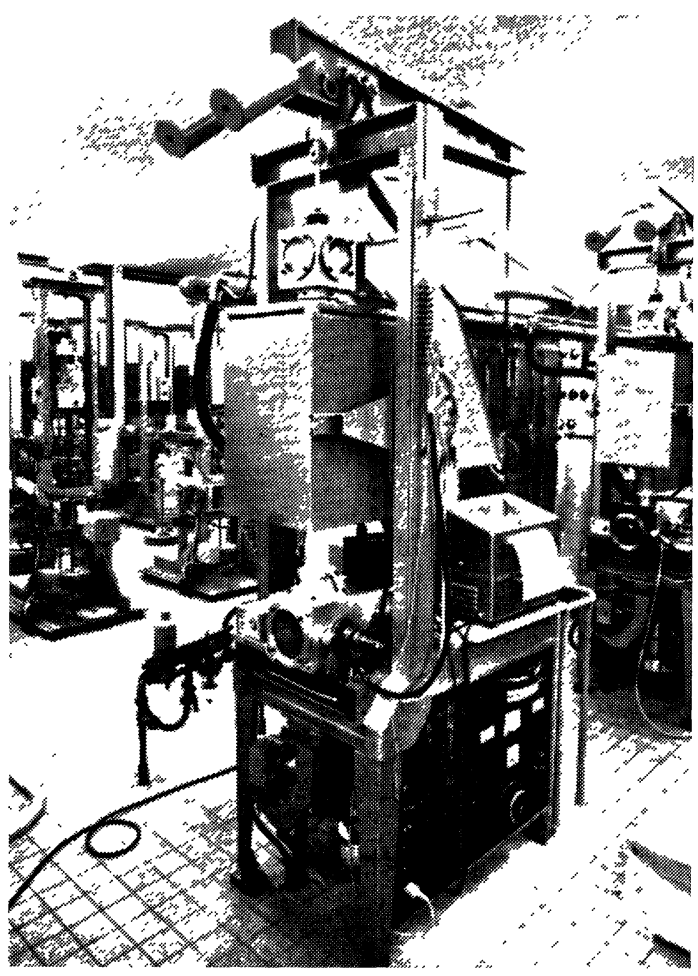

FIG. 4. - Machine de traction et de fluage sous vide.

Essais divers. - Pour terminer les caractéristiques sur matériau nu, mentionnons la mesure du module d'élasticité nécessaire pour le calcul des fréquences de vibration, l'absorption d'énergie sous impact, et la fatigue sur éprouvettes lisses et entaillées.

4.2 CARACTÉRISTIQUES SUR MATÉRIAU PROTÉGÉ. 4.2.1 Oxydation. - Nous avons indiqué plus haut combien il était difficile de rendre compte de tous les régimes moteur, lesquels sont fonction du type d'avion équipé et des missions de celui-ci. Ces régimes peuvent être très différents et se suivre dans un ordre quelconque. Pour compliquer le problème, les alliages base niobium protégés présentent dans les températures intermédiaires de 700 à $900^{\circ} \mathrm{C}$ environ, des phénomènes d'oxydation accélérée. Nous avons constaté, comme beaucoup d'autres laboratoires qu'un maintien court dans le domaine $800^{\circ} \mathrm{C}$ pouvait être plus néfaste pour la suite de l'essai, qu'un maintien isotherme vers $1100^{\circ} \mathrm{C}$ par exemple. Or précisément il y a des parties de la pale de l'aube qui peuvent, en raison des régimes successifs, séjourner alternativement dans ces domaines avec des retours aléatoires à froid. Nous avons donc défini des cycles d'oxydation en laboratoire qui tiennent compte de ces deux cas extrêmes d'oxydation (Fig. 5).

4.2.2 Impact. - Les essais d'impact en laboratoire sont effectués par tir de 2 types de projectiles.

- Projectiles de $0,35 \mathrm{~g}$ à $110 \mathrm{~m} / \mathrm{s}$, donnant de petits impacts ne devant pas apporter de limitation à l'emploi des aubes.
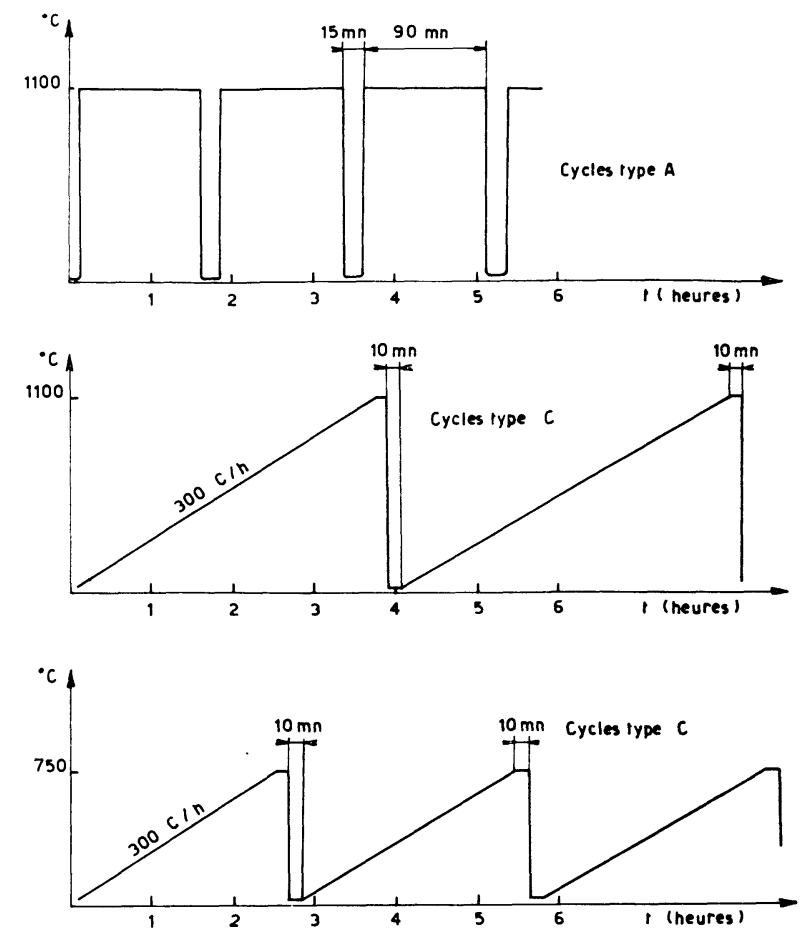

FIG. 5. - Cycles d'essais d'oxydation en thermobalance.

Aube de lurbine $1^{*}$ éloge mobile

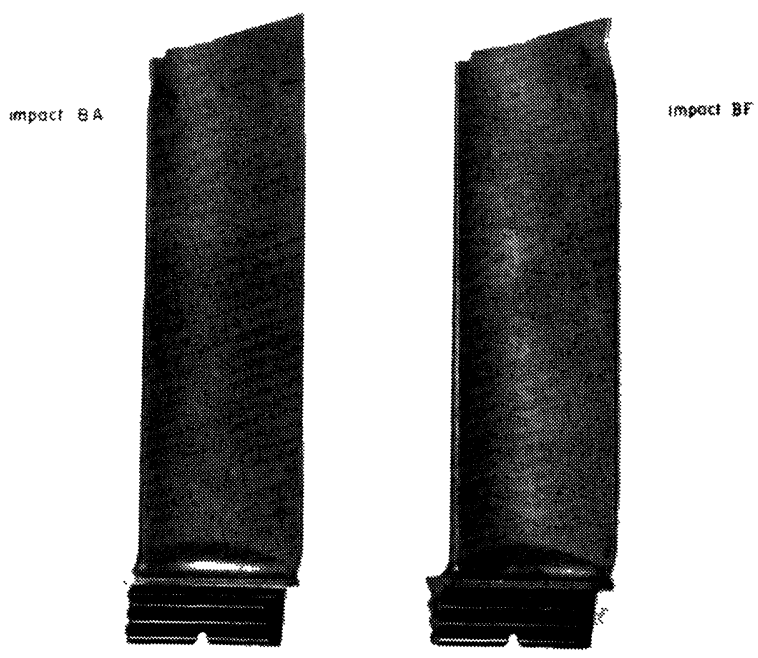

Allioge base niobium protégé

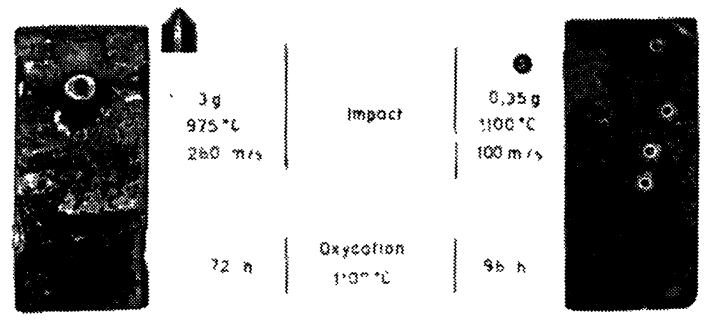

FIG. 6. - Impacts sur moteur et essais en laboratoire. 
- Projectiles de $3 \mathrm{~g}$ à $260 \mathrm{~m} / \mathrm{s}$, correspondant à des impacts ne devant pas conduire à rupture avant la fin d'un vol.

Pour ces essais, nous utilisons une plaquette de $2,5 \mathrm{~mm}$ ou une éprouvette de fatigue thermique (§ 4.2.4) qui a l'avantage de simuler un bord de fuite. Ces éprouvettes peuvent être chauffées pendant l'impact et celui-ci peut être effectué sous un angle normal ou oblique (Fig. 6).

4.2.3 Fluage. - Le fluage sera effectué à l'air sur éprouvettes protégées pour vérifier la tenue globale matériau + protection, l'influence éventuelle du traitement inhérent à la protection et l'aptitude du dépôt à suivre les déformations du substrat.

4.2.4 Fatigue thermique. - Elle est réalisée en laboratoire à l'aide d'un essai à caractère global.

L'appareillage, présenté à la figure 7 , permet de chauffer et de refroidir alternativement le bord mince

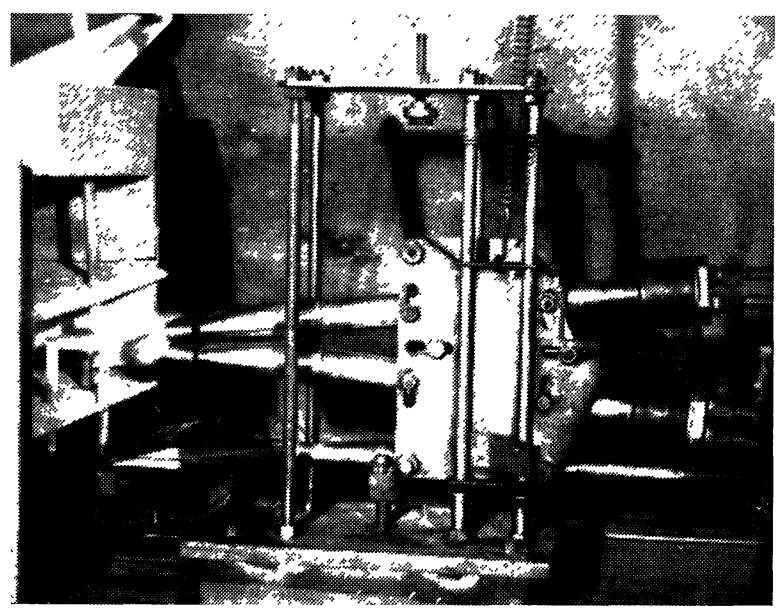

Fig. 7. - Appareil d'essais de fatigue thermique.

d'une éprouvette suivant un cycle défini. L'éprouvette est reproduite à la figure 8 . L'essai consiste à tracer avec 3 ou 4 éprouvettes par famille, les courbes de
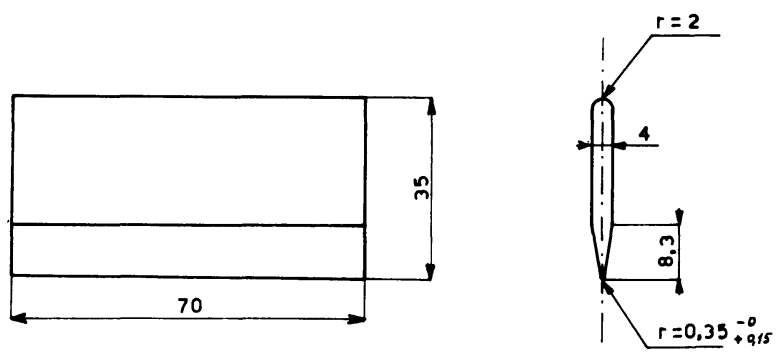

Fig. 8. - Eprouvette de fatigue thermique avant protection

progression des criques en fonction du nombre de cycles. La figure 9 montre des aubes présentant des criques de fatigue thermique.
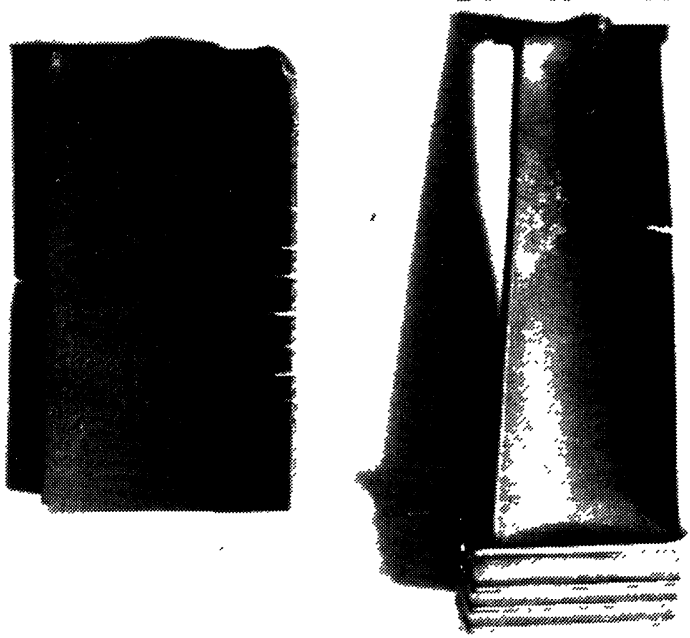

FIG. 9. - Aubes fixe et mobile criquées par fatigue thermique.

On peut donner comme paramètre de tenue en fatigue thermique le terme :

$$
R_{\mathrm{ft}}=\frac{a \cdot \sigma_{r}}{\alpha \cdot E} \quad \text { avec } \quad a=\frac{\lambda}{c \rho}
$$

$a$ : diffusivité thermique;

$c$ : chaleur spécifique ;

$\lambda$ : coefficient de conduction;

$\rho$ : poids spécifique ;

$\sigma_{\mathrm{r}}:$ contrainte de rupture ;

$\alpha$ : coefficient de dilatation;

$E$ : module d'élasticité :

Il faut bien entendu considérer, pour toutes ces caractéristiques, leurs valeurs moyennes dans le domaine de température considéré.

4.2.5 Matage. - L'essai est destiné à vérifier le comportement d'un emmanchement qui simule celui du pied d'aube dans le disque. C'est un essai de fatigue classique en traction répétée, à l'ambiante. Il ne concerne que le dépôt type pied-sapin.

4.2.6 Fatigue lisse et entaillée. - Il est nécessaire de mesurer la limite de fatigue d'éprouvettes protégée et l'influence du dépôt principalement sur la tenue en fatigue sous entaille.

5. Résultats d'essais sur alliages protégés. 5.1 OXYDation. - 5.1.1 Dépôt pied sapin. - Nous avons réalisé pour le pied sapin un dépôt ternaire $\mathrm{Cr}-\mathrm{Ti}-\mathrm{Al}$ ou Ti-Cr-Al en 3 étapes, par des procédés en phase vapeur. Il est destiné à protéger les pieds d'aubes vers $700 / 800^{\circ} \mathrm{C}$ maximum. Il est lisse et mince $(30 \mu)$ et produit un gonflement très faible et régulier. La figure 10 montre quelques résultats. obtenus avec cette couche, d'une part en oxydation isotherme à 700 et $750^{\circ} \mathrm{C}$, d'autre part avec des cycles type $\mathrm{C}$ à $750^{\circ} \mathrm{C}$. On voit qu'on obtient actuellement une durée de vie de $400 \mathrm{~h}$ environ, résultat qui pourra sans doute être amélioré. 

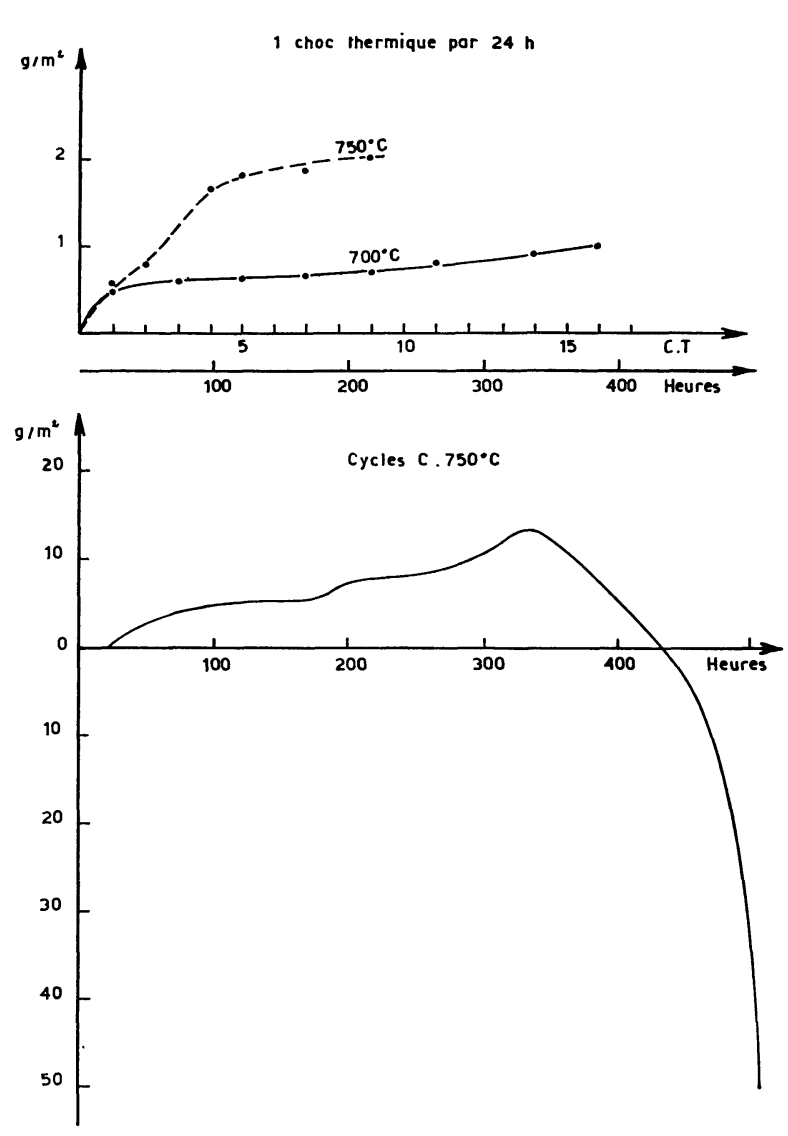

Fig. 10. - Résultats d'essais d'oxydation sur dépôt ternaire chrome-titane-aluminieu en phase vapeur (épaisseur $30 \mu$ ).

5.1.2 Dépôt sur pale. - Ce sont des dépôts à base de siliciures de $\mathrm{Nb}$ avec des éléments tels que chrome, fer, titane avec ou sans étairi-aluminium, tels que ceux mis au point par l'Institut Battelle de Genève.

Nous nous contenterons de donner ci-après des résultats comparés entre cyclage isotherme, période $90 \mathrm{mn}$ à $1100^{\circ} \mathrm{C}$, que nous avons appelé cycle $\mathrm{A}$, et cyclage à montée lente que nous avons appelé cycle $\mathrm{C}$.

\begin{tabular}{ccc} 
Repère & Cycle A à $1100^{\circ} \mathrm{C}$ & Cycle C à $1100^{\circ} \mathrm{C}$ \\
\hline 1 & - & - \\
$2 \mathrm{a}$ & $1200 \mathrm{~h}$ & $50 \mathrm{~h}$ \\
$2 \mathrm{~b}$ & $1000 \mathrm{~h}$ & $120 \mathrm{~h}$ \\
$\mathrm{y}$ & - & $8 \mathrm{~h}$
\end{tabular}

Nous constatons que malgré la durée de séjour à $1100{ }^{\circ} \mathrm{C}, 30$ fois plus courte en cycle $\mathrm{C}$ qu'en cycle $\mathrm{A}$, la durée de vie est divisée par un facteur de l'ordre de 10 à 20 lorsqu'on passe du cycle $\mathrm{A}$ au cycle $\mathrm{C}$.

D'autres essais en cours actuellement permettent cependant d'espérer une amélioration de la tenue en oxydation avec les cycles du type $\mathrm{C}$.
5.2 IмpaCt. - Nous avons fait des impacts de billes de $0,35 \mathrm{~g}$ à $20-850-1100^{\circ} \mathrm{C}$ sous incidence normale et oblique, suivi d'oxydation par cycles de $24 \mathrm{~h}$ à $1100^{\circ} \mathrm{C}$. Dans la plupart des cas, la tenue n'excède pas 48 ou $72 \mathrm{~h}$. La tenue en oxydation est donc fortement diminuée par les impacts.

5.3 Fatigue thermiQue. - Les essais de fatigue thermique effectués sur des éprouvettes protégées n'ont pas mis de problème particulier en évidence. En effet, des dépôts à base de siliciures complexes ne présentaient pas de trace de crique après 600 cycles, ce qui constitue un excellent résultat.

Conclusions. - Dans l'application à des aubes de turbine, il existe actuellement des alliages présentant des performances en caractéristiques mécaniques très intéressantes. Il faut cependant faire attention à certains risques tels que celui d'une température de transition ductile/fragile trop élevée.

Le problème clef est celui de la réalisation d'une protection contre l'oxydation qui présente une fiabilité suffisante dans les conditions particulièrement sévères et diverses de fonctionnement d'un turboréacteur.

Les points critiques semblent être actuellement la tenue à certains cycles thermiques et surtout la tenue aux impacts de corps étrangers, rendue encore plus difficile par la présence de bords de fuite minces. Des résultats encourageants sont obtenus, mais même pour un $2^{\mathrm{e}}$ étage de turbine où la fréquence des impacts est relativement faible, nous ne sommes pas sûrs d'arriver à une solution suffisamment fiable avec les alliages actuels.

La mise au point d'alliages de base, ou d'un premier revêtement épais et ductile, ayant une tenue correcte à l'oxydation d'au moins quelques heures aux températures de fonctionnement, nous paraît importante pour arriver à un minimum raisonnable de fiabilité.

Il est curieux de pouvoir dire qu'un développement plus sûr serait possible dans les turbines à gaz; les problèmes d'impacts sont beaucoup moins sévères sur ces dernières et les cycles thermiques sont bien définis.

Des applications autres que les aubes peuvent être également envisagées sur turboréacteurs, pour des pièces soumises à de très hautes températures, où une grande fiabilité n'est pas aussi impérative : parties de chambre de combustion, anneaux accroche, flamme de post-combustion, etc... En dehors de la résolution du problème technique, celui du prix ne doit pas être oublié et il faudrait que les prix annoncés en production pilote baissent de plus de 10 à 1 pour être acceptables et concurrencer les solutions technologiques plus classiques telles que le refroidissement. 